Introduction Health, safety, wellbeing and work are inextricably linked. In healthcare these are interrelated with patient safety and quality of experience, care and outcomes. The World Health Organisation (WHO) identifies that creating 'Healthy Workplaces' is the right, legal and smart thing to do (2010). What importance is given to this in healthcare?

Methods A recent Masters studies in a large healthcare facility revealed an abundance of evidence of the critical relationships in creating healthy workplaces. These were reinforced through a ground level qualitative audit and ongoing work. They were, aligned with a Maori cultural perspective of health, Te Whare Tapa Wha and the WHO 'Healthy Workplaces' definition and action model.

Result Through onoging collaborative work, using an enviromental scan and gap analysis, we have developed a three year strategic, quality improvement strategy with fifteen aims. The thematic analysis from the study is guiding our journey.

Discussion Pscychosocial risks are noted as being one of the greatest health and safety challenges of the modern day workplace. Do we have enough focus or understanding on these and how to manage them? What are the effects on healthcare workers?

The international health sector is seeing signs of increasing burnout, stress, moral distress, emotional exhaustion and increasing reports of bullying and harrassment. Additionally it has an ageing workforce; known effects on shiftworkers; increasing long term conditions inclusive of poor mental health; new ways of working; changing technology and people having to do more with less. These are already impacting on workers and workplaces.

Where does Occupational Health and Safety fit in? What are we doing and is it time to bridge the gap with more connected, collaborative approaches with public health, organisational health and others? We cannot afford to ignore the critical interfaces, as the cost of inaction is high at many levels.

\section{SHARP DEVICES INJURIES AMONG RESIDENT PHYSICIANS IN ISMAILIA HOSPITALS}

Ayman Ekram Fahim. Occupational Health Unit, Department of Community, Environment and Occupational Medicine, Suez Canal University, Ismailia 41522, Egypt

\subsection{6/oemed-2018-ICOHabstracts.988}

Introduction Injuries to health-care workers from needle stick and other sharps carry significant risks of transmitting blood borne pathogens such as HBV, HCV and HIV, with serious consequences. The aim was to determine the incidence and assess the context of Exposure Incidents (EI) among residents and nurses at Suez Canal University hospital.

Methods A self-administered questionnaire was distributed to 217 residents who were less than 3 years of work/training experience. The questionnaire included details of exposure incidents of high risk and low risk exposures. 'high risk' exposure includes; a penetrating injury caused by a needle or other sharp object, and causing visible bleeding. 'low risk' as (a) a slight, superficial abrasion caused by a needle or other sharp object without obvious bleeding or (b) an existing skin wound coming into direct contact with blood or other body fluids. Also, activities/procedures leading to exposure incidents, and preventive measures used were recoded. All participants had informed consent.
Results One hundred seventy one (78.8\% of the participants) responded to the questionnaire, 117 workers (68.4.8\%) reported to have (EI) (either one or more), and two hundred and thirty seven (EI) were recorded over 12 months with corresponding incidence of 0.68 and 2.1 (EIs) per worker/year. Seventy seven of exposure incidents (32.4\%) were considered ' high risk', while $67.6 \%$ were 'low risk'. Injection needles were the most common device $(53.7 \%$ of exposure episodes) causing (EI) followed by suture needles (37.8\%). Phlebotomy/ injection and suturing were the most common procedures of exposure episodes $(44.7 \%$ and $39.1 \%$, respectively). Workers always recap needles/IV-catheters $(47.1 \%)$ after use, and only (7.9\%) always uses PPE during procedures.

Conclusion Sharp device injuries are common among physicians in their early years of work/training, and are often not reported or lack a reporting system. Improved prevention/ safety practices and reporting strategies are needed.

\section{WORK-RELATED PSYCHOSOCIAL AND PHYSICAL RISK FACTORS AND UPPER LIMB DISORDERS IN HAND- INTENSIVE HEALTH CARE WORKERS - AN APPLIED EPIDEMIOLOGICAL PERSPECTIVE}

${ }^{1}$ Birgit A Greiner*, ${ }^{1,2}$ Sheilah Nolan 'Dervla A Hogan. 'University College Cork, Dep. Epidemiology and Public Health, Cork, Ireland; ${ }^{2}$ Kerry Health and Safety, Killarney, Ireland

\subsection{6/oemed-2018-ICOHabstracts.989}

Introduction Musculoskeletal injuries are common in healthcare workers. Research and prevention have been focussing on back injuries, the scientific evidence on work-related upper limb disorders (WRULDs) is scarce. Physical and psychosocial work exposures are considered as relevant in the aetiology. Hand-intensive health care occupations, e.g. physiotherapists are commonly exposed to physical risk factors including repetitive movements while applying force and sustained awkward positions. The objective was to investigate associations between physical and psychosocial work exposures and ULDs while adjusting for non-work-related explanations.

Methods Cross-sectional with 347 Irish Chartered Physiotherapists, Physical and Athletics Therapists in hospitals and private practice (proportionate cluster and random sampling). Participants completed questions about neck, shoulder, elbow, wrist, thumb and finger symptoms (Nordic Questionnaire); psychosocial work exposures (COPSOQ), rest breaks, scheduling, physical work load, Physical Exertion (Borg scale), lifestyle and mental health. Logistic regression with psychosocial and physical factors and ULDs with adjustment for lifestyle-related issues (bmi, smoking), depression (GHQ) and leisure time injury.

Result Work tempo $(\mathrm{OR}=1.17)$, predictability $(\mathrm{OR}=0.76)$, peer support $(\mathrm{OR}=0.81)$ and supervisory support $(\mathrm{OR}=0.71)$ were significantly associated with UL symptoms in the past 12 months, work predictability $(\mathrm{OR}=0.82)$, influence at work $(\mathrm{OR}=0.91)$, supervisory support $(\mathrm{OR}=0.81)$ and peer support $(\mathrm{OR}=0.77)$ were significantly associated with incapacitating symptoms. Therapists who did not schedule their appointments were twice as likely for ULDs in a least one body site $(\mathrm{OR}=2.3)$, those with rests breaks below $5 \mathrm{~min}$ after each treatment were at increased odds for incapacitating symptoms. $(\mathrm{OR}=2.3)$, physical exertion and repetitive movements were associated with 12 month prevalence $(\mathrm{OR}=1.3)$. All analyses adjusted for confounders. 